\title{
Progressive Multiple Sclerosis Transcriptome Deconvolution Indicates Increased M2 Macrophages in Inactive Lesions
}

\author{
Sai Batchu \\ National Heart, Lung, and Blood Institute (NHLBI), National Institutes of Health (NIH), Bethesda, MD, USA
}

\section{Keywords}

Multiple sclerosis · Macrophage $\cdot$ RNA-Sequencing $\cdot$ Lesion

\begin{abstract}
Accumulating evidence suggests M2 macrophages contribute to tissue reparation and limit inflammation in multiple sclerosis (MS). However, most studies have focused on murine models without substantial support through human MS observations. The present study aimed to quantify the relative abundances of M2 macrophages in different lesion types excised from human MS patients. CIBERSORTx, an established RNA deconvolution algorithm, was applied on bulk RNA-sequencing data developed from 98 lesions from 10 progressive MS patients and 5 neuropathological control donors. A validated gene signature matrix for 22 human hematopoietic cell subsets was used to infer the relative proportions of immune cells that were present in the original lesion. Deconvolution of the bulk gene expression data showed that inactive lesions contained significantly more M2 macrophages compared to normal white matter control samples. The findings suggest that M2 macrophages may play a role during lesion inactivity in MS.
\end{abstract}

(c) 2020 S. Karger AG, Basel

\section{Introduction}

Multiple sclerosis (MS) is a heterogeneous autoimmune disease characterized by demyelination and neurodegeneration [1]. As the disease advances, pathological changes are observed in the brain white matter (WM) that can be categorized into distinct lesion types based on measures of myelin loss and immune infiltrate [2]. While initial studies have focused on the role of $\mathrm{T}$ cell subsets in MS $[3,4]$, emerging evidence has implicated macrophages as well [5]. Recent discussions have centered on the role of M2 macrophages (also known as regulatory or alternatively activated macrophages) in MS, with evidence for these myeloid subsets being involved of pro-repair action and lesion resolution $[5,6]$. Yet, most studies implicating M2 macrophages have examined the experimental autoimmune encephalomyelitis animal model $[7,8]$ with less evidence cited in human MS [9]. To address this gap, the present study aimed to quantify the relative abundances of M2 macrophages in different lesion types excised from progressive human MS brain WM through transcriptomic deconvolution.

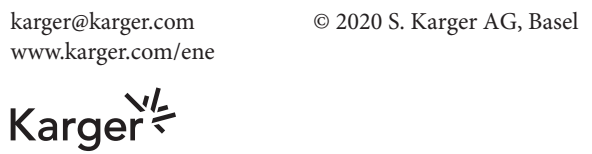




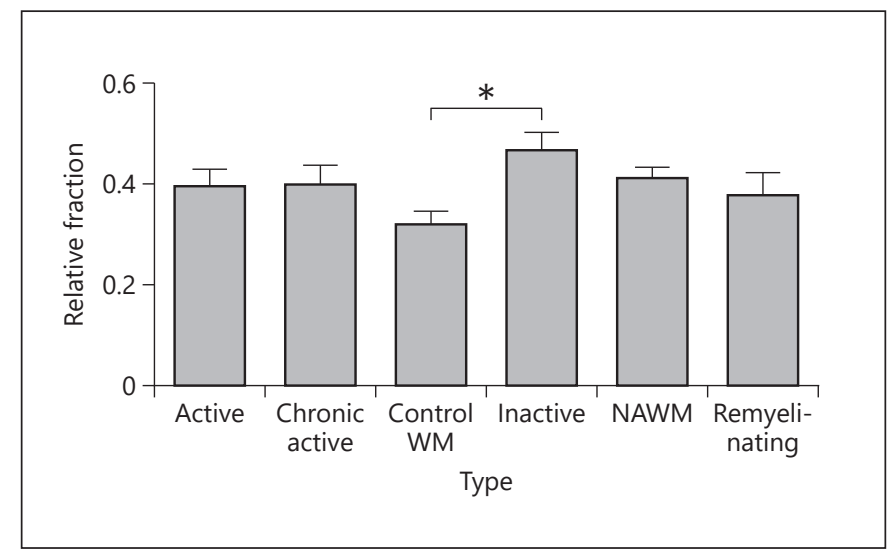

Fig. 1. M2 macrophage relative abundances in MS lesion types; mean \pm SEM. ${ }^{*} p<0.05$. MS, multiple sclerosis; WM, white matter; NAWM, normal-appearing white matter.

\section{Materials and Methods}

A previously published gene expression dataset consisting of brain WM areas from 10 progressive human MS and 5 neuropathological control subjects was used [10]. The dataset consists of bulk RNA-sequencing gene expression data for $25 \mathrm{WM}$ control samples, 19 normal appearing WM, 6 remyelinating, 18 active, 13 inactive, and 17 chronic active lesion samples. The data were generated using Illumina NextSeq550 platform and was downloaded from the Gene Expression Omnibus database (http://www.ncbi. nlm.nih.gov/geo) under accession GSE138614.

CIBERSORTx, an established machine-learning RNA deconvolution algorithm, was implemented to provide an estimation of the relative abundances of immune cells from the original lesions [11]. The algorithm accurately quantifies the relative fractions of distinct cell types within a complex gene expression mixture given a priori cell type-specific gene signatures. Specifically, the algorithm requires (1) a matrix of bulk tissue gene expression profiles to be deconvoluted and (2) a signature matrix containing signature gene expression profiles for cell subsets of interest. To estimate the proportion of a specific cell type, the algorithm assesses the relative expression changes of signature genes related to the cell type of interest compared with the expression of all other genes in the sample [11].

For the present study, a validated microarray-derived signature matrix containing a total of 547 genes for distinguishing 22 human hematopoietic cell subsets, termed "LM22," was used to deconvolute the bulk lesion tissue gene mixture matrix [11]. The CIBERSORTx algorithm was run with bulk-mode batch correction and 500 permutations in the relative mode. Quantile normalization was not implemented as dataset was generated from RNA-sequencing. Deconvoluted samples were deemed significant if CIBERSORTx $p$ value $<0.05$, which represents the significance of the deconvolution results across all cell subsets for goodness-of-fit [11].

The data output from CIBERSORTx was downloaded and analyzed with R programming language. One-way ANOVA was applied to analyze differences between lesion types followed by Tukey's HSD post hoc test. Results presented as the mean \pm SEM. $p$ value $<0.05$ was deemed significant.

\section{Results}

After deconvolution, all 98 samples were significant at the criteria for CIBERSORTx $p$ value $<0.05$ and were deemed suitable for further analysis. Comparing M2 macrophage fractions across lesion types showed that inactive lesions exhibited a significantly higher relative abundance than control WM samples (shown in Fig. 1). The algorithm estimated the relative fractions of these cell types through unique signature genes specific to M2 macrophages taken from the input LM22 signature matrix (see online suppl. Table 1; for all online suppl. material, see www.karger.com/doi/10.1159/000510075) [11]. Significant changes in the relative proportions were not observed among the other 21 immune cell subtypes evaluated, including related subtypes such as monocytes and M1 macrophages (shown in online suppl. Fig. 1).

\section{Discussion}

As presented here, the digital cytometric technique has shown a significantly increased M2 macrophage abundance in inactive MS lesions compared to control $\mathrm{WM}$. This finding implies a potential role for these alternatively polarized macrophage type during lesion inactivity, which is characterized by demarcated areas of demyelination with little to no inflammatory activity [2]. Previously, Giles et al. [12] found an increased presence of cells expressing mannose receptor (CD206), a marker of alternative $\mathrm{M} 2$ polarization, near inactive lesion centers in murine experimental autoimmune encephalomyelitis. Altogether, the evidence suggests M2 macrophages may contribute to resolution of lesion inflammation. Surprisingly, no significant difference was seen with remyelinating lesions, possibly attributing to the small sample size.

A limitation of the current study is the in silico approach to deconvolute gene expression data from bulk tissue sample. The data processing does not account for the spatial relationship of tissue and cytoarchitecture. Additionally, further validation is warranted to differentiate between microglia and macrophage subsets, as certain signature genes may be shared between these cell types. Nonetheless, this digital cytometric technique has previously replicated conventional flow cytometry data with significant clinical implications [13-16].

Based on the hypothesis-generating data presented here, further studies are warranted to elucidate the role of M2 macrophages in MS. In conclusion, the digital ap- 
proach used herein implicated M2 macrophages in inactive lesions, suggesting that this myeloid subtype may play a role in human MS.

\section{Statement of Ethics}

This article does not contain any studies with human participants performed by the authors and no ethical approval was needed. All the data used in the study were already available online and published in a previous study, which is cited in the references. Our research complies with the guidelines for human studies and was conducted ethically in accordance with the World Medical Association Declaration of Helsinki.

\section{Conflict of Interest Statement}

The author has no conflicts of interest to declare.

\section{Funding Sources}

The present work was partly supported by the intramural research program of the National Heart, Lung, and Blood Institute (NHLBI), the National Institutes of Health (NIH), Grant No. Z99 HL999999. The content is solely the responsibility of the author and does not necessarily represent the official views of the NIH.

\section{References}

1 Nally FK, De Santi C, McCoy CE. Nanomodulation of macrophages in multiple sclerosis. Cells. 2019 Jun;8(6):543.

2 Reynolds R, Roncaroli F, Nicholas R, Radotra $B$, Gveric D, Howell O. The neuropathological basis of clinical progression in multiple sclerosis. Acta Neuropathol. 2011 May; 122(2): 155-70

3 McFarland HF, Martin R. Multiple sclerosis: a complicated picture of autoimmunity. Nat Immunol. 2007 Sep;8(9):913-9.

4 Fletcher JM, Lalor SJ, Sweeney CM, Tubridy $\mathrm{N}$, Mills KH. T cells in multiple Sslerosis and experimental autoimmune encephalomyelitis. Clin Exp Immunol. 2010 Oct;162(1):1-11.

5 Mishra MK, Yong VW. Myeloid cells: targets of medication in multiple sclerosis. Nat Rev Neurol. 2016 Aug;12(9):539-51.

6 Mikita J, Dubourdieu-Cassagno N, Deloire MS, Vekris A, Biran M, Raffard G, et al. Altered M1/M2 activation patterns of monocytes in severe relapsing experimental rat model of multiple sclerosis. Amelioration of clinical status by M2 activated monocyte administration. Mult Scler. 2011 Sep;17(1):215.
7 Tierney JB, Kharkrang M, La Flamme AC. Type II-activated macrophages suppress the development of experimental autoimmune encephalomyelitis. Immunol Cell Biol. 2009; 87(3):235-40

8 Jiang HR, Milovanović M, Allan D, Niedbala W, Besnard AG, Fukada SY, et al. IL-33 attenuates EAE by suppressing IL-17 and IFN- $\gamma$ production and inducing alternatively activated macrophages. Eur J Immunol. 2012; 42(7):1804-14

9 Raine CS. Multiple sclerosis: the resolving lesion revealed. J Neuroimmunol. 2017 Mar; 304:2-6.

10 Elkjaer ML, Frisch T, Reynolds R, Kacprowski T, Burton M, Kruse TA, et al. Molecular signature of different lesion types in the brain white matter of patients with progressive multiple sclerosis. Acta Neuropathol Commun. 2019 Dec;7(1):205

11 Newman AM, Steen CB, Liu CL, Gentles AJ, Chaudhuri AA, Scherer F, et al. Determining cell type abundance and expression from bulk tissues with digital cytometry. Nat Biotechnol. 2019 Jul;37(7):773-82.
12 Giles DA, Washnock-Schmid JM, Duncker PC, Dahlawi S, Ponath G, Pitt D, et al. Myeloid cell plasticity in the evolution of central nervous system autoimmunity. Ann Neurol. 2018;83(1):131-41.

13 Chen B, Khodadoust MS, Liu CL, Newman AM, Alizadeh AA. Profiling tumor infiltrating immune cells with CIBERSORT. Methods Mol Biol. 2018 Jan;1711:243-59.

14 Rohr-Udilova N, Klinglmüller F, SchulteHermann R, Stift J, Herac M, Salzmann M, et al. Deviations of the immune cell landscape between healthy liver and hepatocellular carcinoma. Sci Rep. 2018 April;8(1): 6220.

15 Tang G, Yin W. Development of an immune infiltration-related prognostic scoring system based on the genomic landscape analysis of glioblastoma multiforme. Front Oncol. 2020 Feb;10:154

16 Yang S, Liu T, Cheng Y, Bai Y, Liang G. Immune cell infiltration as a biomarker for the diagnosis and prognosis of digestive system cancer. Cancer Sci. 2019 Oct;110(12):3639_ 49. 